\author{
Associate Professor Alexandra TKACENKO, PhD \\ The State University of Moldova \\ E-mail: alexandratkacenko@gmail.com
}

\title{
THE METHOD OF SYNTHESIS FUNCTIONS FOR SOLVING THE MULTI-CRITERIA LINEAR-FRACTIONAL TRANSPORTATION PROBLEM WITH "BOTTLENECK" CRITERION
}

\begin{abstract}
In the paper, it is developed an adapted version of Jose A. Diaz's algorithm [2] for solving the multi-criteria linear-fractional transportation problem with the same "bottleneck" denominators, additionally the same time "bottleneck" criterion is including separately. It generates for each (feasible) time value the best compromise multi criteria solution, which is situated closely of ideal solution. So, finally, we will obtain one finite set of function-distance optimal compromise solutions, each corresponding to one time level solutions for solving the multi-criteria fractional transportation problem with the same "bottleneck" denominators, separately including the time "bottleneck" criterion. The proposed algorithm has been tested on several examples and proved to be quite effective.
\end{abstract}

Keywords: Fractional multi-criteria transportation model, "bottleneck" criterion, efficient solution, a best compromise solution, function-distance optimal compromise solutions.

\section{JEL Classification: C44, C61}

\section{Introduction}

The interest of the multi- criteria optimization problem is on the rise, including the multi-criteria transportation model, which has numerous practical applications. The efficient solutions of the multi-criteria transportation problem of liner type can be achieved using various algorithms developed in [2], [5, [6], [8], [10] and many others. A large variety of algorithms have been proposed for different kinds of multi-criteria transportation problems of "bottleneck" type [1], [3], [4]. In [1] for solving the three-criteria transportation problem, including the "bottleneck" Aneja and Nair developed an efficient algorithm, Wild and Karwan in [11] proposed an efficient algorithm for solving the generalized $r$-criteria transportation problem of the same type. The efficient algorithms for solving one-

DOI: $10.24818 / 18423264 / 53.1 .19 .10$ 
criterion fractional transportation model of "bottleneck" type are proposed by Sharma and Swarup in [7] and by Tkacenko in [9] for multi-criteria fractional transportation model of the same type. Frequently, the decision maker proposes to find a single solution for the multicriterial issue, which would achieve the best compromise between criteria, on the grounds that he does not have time to analyse the entire set of efficient solutions. In this paper we propose an algorithm based on the method of synthesis functions, leading to an optimal compromise solution for multi-criteria fractional transport model of bottleneck type.

\section{Problem formulation}

The mathematical model of the multi-criteria fractional transportation problem of "bottleneck" with deterministic data is the following:

$$
\begin{aligned}
& \min F_{1}=\frac{\sum_{i=1}^{m} \sum_{j=1}^{n} c_{i j}{ }^{1} x_{i j}}{\max _{i, j}\left\{t_{i j} \mid x_{i j}>0\right\}} \cdots \ldots \min F_{r}=\frac{\sum_{i=1}^{m} \sum_{j=1}^{n} c_{i j}{ }^{r} x_{i j}}{\max _{i, j}\left\{t_{i j} \mid x_{i j}>0\right\}} \\
& \min F_{r+1}=\max _{i, j}\left\{t_{i, j} \mid x_{i, j}>0\right\} \quad \text { in conditions }: \\
& \sum_{j=1}^{n} x_{i j}=a_{i}, \quad(\forall) i=\overline{1, m}, \sum_{i=1}^{m} x_{i j}=b_{j}, \quad(\forall) j=\overline{1, n} \\
& \sum_{i=1}^{m} a_{i}=\sum_{j=1}^{n} b_{j}, \quad x_{i j} \geq 0, i=\overline{1, m}, \quad j=\overline{1, n}
\end{aligned}
$$

where: $c_{i j}-\cos t$ of transportation of a unit of hom ogeneous product from the sourse $i$ to destination $j ; a_{i}$-availability of sourse $i ; b_{j}-$ requirement of destination $j ; x_{i j}$-amount transported from $i$ to $j$; $t_{i j}$-time of transporting a unit from sourse i to destination $j$;

We can observe, that in model (1)-(2) the first " $r$ " criteria is of linearfractional type, moreover, with identical denominators. The denominator function appears again as a separate the $(r+1)$ criterion, being a time-constraining criterion, met in special literature as a bottleneck-type criterion. Particular cases of the model (1)-(2) were analysed in [9]. In 10, the author proposes an algorithm that records for each time value all the efficient solutions for model (1) - (2), so in the end, the decider has the set of all the efficient solutions of the model. 
The Method of Synthesis Functions for Solving the Multi-criteria Linear-fractional Transportation Problem with "Bottleneck" Criterion

\section{Theoretical considerations}

In order to investigate the model of multiple criteria, we should propose firstly the definition of efficient solution for the deterministic model (1)-(2).

Let suppose that: $(\overline{\mathrm{X}}, \overline{\mathrm{T}})$ is one basic solution for the model (1)-(2), where: $\overline{\mathrm{T}}=\max _{i, j}\left\{\bar{t}_{i j} / \bar{x}_{i j}>0\right\}$.

Definition 1. The basic solution $(\bar{X}, \bar{T})$ of the model $(1)-(2)$ is a basic efficient one if and only for any other basic solution $(X, T) \neq(\bar{X}, \bar{T})$ for which exists at least one index $j_{1} \in(1, \ldots r)$ for which the relation: $F_{j_{1}}(X) \leq F_{j_{1}}(\bar{X})$ is true, there immediately exists another, at least, one index $\exists j_{2} \in(1, \ldots . r)$, where $j_{2} \neq j_{1}$, for which at least, one of the both relations $F_{j_{2}}(\bar{X})<F_{j_{2}}(X)$ or $\bar{T}<T$ is true. If all of these three inequalities are verified simultaneously with the equal sign, it means that the solution is not unique.

Definition 2. The basic solution $(\overline{\mathrm{X}}, \overline{\mathrm{T}})$ of the model $(1)-(2)$ is one of the optimal compromise solution for a certain time $\bar{T}$, if its corresponding basic solution $\overline{\mathrm{X}}$ is located closest to the optimal solutions for the first $r$ criteria.

We will consider the next multiple-criteria transportation model of "bottleneck" type:

$$
\begin{aligned}
& \min F_{1}=\sum_{i=1}^{m} \sum_{j=1}^{n} c_{i j}^{1} x_{i j}, \quad \min F_{2}=\sum_{i=1}^{m} \sum_{j=1}^{n} c_{i j}^{2} x_{i j} \ldots \ldots \ldots . . \\
& \min F_{r}=\sum_{i=1}^{m} \sum_{j=1}^{n} c_{i j}^{r} x_{i j}, \min F_{r+1}=\max _{i, j}\left\{t_{i, j} \mid x_{i, j}>0\right\}
\end{aligned}
$$

in the same conditions like in (2).

Analysing the model (1) - (2) and (3), it is evident, that both models have the same set of basic solutions, because of the same availability domains. 
Definition 3. The basic solution $X^{+}$of the model (3) is considered idel for its first $r$ criteria if for it the relationship are true $F_{k}^{+}\left(X^{+}\right)=\min _{X \in D} F_{k}(X), k=\overline{1, r}$, where $Z^{+}=\left(F_{1}^{+}, F_{2}^{+}, \ldots, F_{r}^{+}\right)$is the vectorof the values of the objective functions in the ideal solution, but $D$ is the admissible domain described by the relationship (2).

Theorem 1. [9] The set of the basic efficient solutions of the model (1)-(2) and (3) coincide.

Demonstration of the theorem is brought to [9].

Obviously, the set of the basic best optimal compromise solutions of model (1) (2) and (3) also coincide.

Theorem 2. Any best compromise solution for model (3) is also efficient for this. Proof. Let suppose, that in time matrix - $T$ of model (3) the time value is ordered in ascending order, thus we will assign the matrix cells of the time (for identical values of $t$, we will consider different, increasing indices) the indices obtained in the result of its ordination. So, the total number of indices will be: $k=m \cdot n$. We will consider at first iteration at least $m+n-1$ cells used, and we will assign $k_{1}=m+n-1$;

We'll calculate $\overline{\mathrm{T}}_{1}=\max _{i, j}\left\{\bar{t}_{i j} / \bar{x}_{i j}>0\right\} \quad$ of matrix $T$, for which $\exists \bar{X}_{1} \in D$ (D is the domain of admissible solutions for model (3), described by relations (2)), that is of the best compromise solution for the first $r$ criteria of model (3). We will consider the cells for which we have true the relationship $t_{i j}>\bar{T}_{1}$, as blocked. According the above definition, result immediately Solution $\bar{X}_{1}$ register the next values of objective function (first $r$ criteria):

$$
\left(F_{1}\left(\bar{X}_{1}\right), F_{2}\left(\bar{X}_{1}\right) \ldots F_{r}\left(\bar{X}_{1}\right)\right) \text {; }
$$

We assume that solution $\bar{X}_{1}$ is not efficient for the corresponding $\overline{\mathrm{T}}_{1}$. In accordance with Definition 1 immediately apparent that, for the same time value $\bar{T}_{1}$, there is another basic solution, different from the first, $X \neq \bar{X}_{1}$ for which exists at least one index $j_{1} \in(1, \ldots r)$ for which the relation $F_{j_{1}}(\mathrm{X})<\left\langle F_{j_{1}}(\overline{\mathrm{X}})\right.$ is true. In this case, obviously, the distance from point $X$ to the ideal point will be smaller than from $\bar{X}_{1}$ to this, calculated for any metric. In this case, obviously, 
The Method of Synthesis Functions for Solving the Multi-criteria Linear-fractional Transportation Problem with "Bottleneck" Criterion

the distance from point $X$ to the ideal point will be smaller, calculated for any metric. This contradicts the assumption that $\bar{X}_{1}$ achieves the best compromise for the first $r$ criteria of the model (3). Therefore, the solution $\bar{X}_{1}$ is also efficient for the model (3). The similar procedure is applied to the following unblocked $T$ cells until all of cells have been unlocked, and for each unlocked time cell, we will highlight its corresponding solution, that achieves the best compromise for the first $\mathrm{r}$ criteria of the model (3). Finally, for model (3) we will have the set of solutions that make the best compromise of the first $r$ criteria for any time value from the $\mathrm{T}$ matrix.

Remark 1. The set of basic efficient solutions and of basic best optimal compromise solutions of model (1) - (2) and (3) coincide.

Theorem 3. The set of the best compromise solution of model (1)-(2) and (3) coincide.

Proof. Let suppose that we have determined the set of the best compromise solutions, each solution being associated with the corresponding time of its realization, for the model (3), which is the next:

$\left\{\left(X_{1}, T_{1}\right),\left(X_{2}, T_{2}\right), \ldots,\left(X_{p}, T_{p}\right)\right\}$; We will consider for $T_{1}$ the record vector of the first $r$ criteria of model (3): $\left\{F_{1}, F_{2}, \ldots, F_{r}\right\}$. These denote the closest placement of the basic solution $X_{1}$ by the ideal solution for time $T_{1}$ for any distance measurement metric. We will assume that $X_{1}$ is not the solution of the best compromise for model (1) - (2), corresponding to time $T_{1}$. In this case, there is another $\tilde{X}_{1}$ solution so that $\tilde{X}_{1} \neq X_{1}$ and will be placed closer to the ideal solution than the first. The record vector of the first $r$ criteria of model (1)-(2) is the following: $\left\{\frac{\tilde{F}_{1}}{T_{1}}, \frac{\tilde{F}_{2}}{T_{1}}, \ldots, \frac{\tilde{F}_{r}}{T_{1}}\right\}$. Since $T_{1}$ is the same for all $\mathrm{r}$ criteria of model (1) (2), it can be considered as a constant, which does not influence the value of the distance. Obviously, the distance is only influenced by the record: $\left\{\widetilde{F}_{1}, \widetilde{F}_{2}, \ldots, \tilde{F}_{r}\right\}$.Since $X_{1}$ is not efficient for the model (1)-(2), it results that the distance of the $\left\{F_{1}, F_{2}, \ldots, F_{r}\right\}$ records to the ideal point is less than the distance evaluated for the $\left\{\widetilde{F}_{1}, \widetilde{F}_{2}, \ldots, \tilde{F}_{r}\right\}$ records, which contradicts the assumption that 
$X_{1}$ is the best compromise solution for the model (3), corresponding to the time $T_{1}$. Therefore, $X_{1}$ is also the solution of the best compromise for model (1) - (2), corresponding to time $T_{1}$. Analogously, the claim is made for the other values of time. Therefore, the multitude of the best compromise solutions of the model (3) is necessarily of the best compromise and for model (1) - (2). Similarly, it demonstrates that the set of the best compromise solutions for the model (1) - (2) is of the best compromise for the model (3). Therefore, the multitudes of the best compromise solutions for models (1) - (2) and (3) coincide, what had to be demonstrated

\section{Method of L-compromise [2]}

We will consider a multi criteria transport model of the linear type represented by the first $r$ criteria of model (3) and domain restrictions (2). It is the next:

$$
\begin{aligned}
& \min F_{1}=\sum_{i=1}^{m} \sum_{j=1}^{n} c_{i j}^{1} x_{i j}, \min F_{2}=\sum_{i=1}^{m} \sum_{j=1}^{n} c_{i j}^{2} x_{i j}, \ldots, \min F_{r}=\sum_{i=1}^{m} \sum_{j=1}^{n} c_{i j}^{r} x_{i j}, \\
& \sum_{j=1}^{n} x_{i j}=a_{i}, \quad(\forall) i=\overline{1, m}, \quad \sum_{i=1}^{m} x_{i j}=b_{j}, \quad(\forall) j=\overline{1, n} \\
& \sum_{i=1}^{m} a_{i}=\sum_{j=1}^{n} b_{j}, \quad x_{i j} \geq 0, \quad i=\overline{1, m}, \quad j=\overline{1, n}
\end{aligned}
$$

\section{Definition 4.}

The basic solution $X^{*}$ of $\bmod$ el (3) is considered of function-

dis $\tan$ ce optimal compromise for its first $r$ criteria and fixed $T$ if for it is true the relationship $\min _{X \in D} \sum_{k=1}^{r} \frac{F_{k}(X)}{F_{k}^{+}}=\sum_{k=1}^{r} \frac{F_{k}\left(X^{*}\right)}{F_{k}^{+}}$for the cells with time value $: t_{i j} \leq T$, where: $F_{k}^{+}=\min _{X \in D} F_{k}(X)$, for $k=1, \ldots, r$. 
The Method of Synthesis Functions for Solving the Multi-criteria Linear-fractional Transportation Problem with "Bottleneck" Criterion

We will use a linear approximation to measure the distance between the permissible solution and the ideal one. We will define the new variables as follows:

$$
Y_{k}=\frac{1}{F_{k}^{+}} \sum_{i=1}^{m} \sum_{j=1}^{n} c_{i j}^{k} x_{i j}, \quad k=1, \ldots, r
$$

From formula (6) it follows that for any values $(\forall) X \in D$, the corresponding values of $Y_{k}$, are obviously $\geq 1$; and if $Y_{k}=1$, then the corresponding solution $X$ is optimal for criterion $k$;

We will build the following function:

$$
F(X)=\sum_{k=1}^{r} Y_{k},
$$

which is a function of measuring the degree of touch of optimality, obviously being of linear type. It is clear that min $F(X)$ possible may be equal to $r$ and this is also achieved if the problem admits an ideal solution, i.e. if we have:

$$
\min F(X)=r \text {. }
$$

In other cases, we will have a true relationship: $F(X)>r$.

Thus, the mathematical model of the proposed problem is the following:

$$
\min \sum_{k=1}^{r} Y_{k}
$$

in condition (5)-(6)

We can observe that the model (9) is a one criterion transportation model of linear type.

Definition 4. The optimalbasic solution $X_{L}^{+}$of the model (9) is considered a $L$-compromise solution for the model (4)-(6).

Theorem 4. Any L- compromise solution for model (4)-(6) is also efficient for this.

Proof. The demonstration we will do it, assuming the contrary.

Let: $X_{L}^{+}$- L-compromise solution of the model (4)-(6), which is not efficient for it.

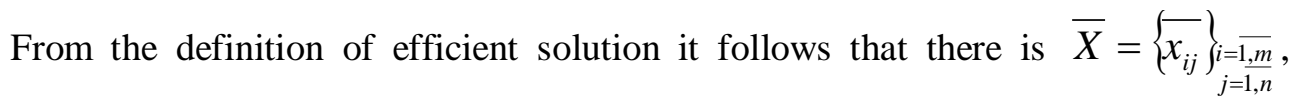
$\bar{X} \in D$ și $\overline{X \neq} X_{L}^{+}$so that the relations are true:

$$
\sum_{i=1}^{m} \sum_{j=1}^{n} c_{i j}^{k} \overline{x_{i j}} \leq \sum_{i=1}^{m} \sum_{j=1}^{n} c_{i j}^{k} x_{i j}^{+}, \quad k=\overline{1, r}
$$


and at least for some indices the inequality (10) is strict, i.e. for them is true the relation:

$$
\sum_{i=1}^{m} \sum_{j=1}^{n} c_{i j}^{k} \overline{x_{i j}} \neq \sum_{i=1}^{m} \sum_{j=1}^{n} c_{i j}^{k} x_{i j}^{+} .
$$

In this case we have the following relationship true:

$$
\frac{1}{F_{k}^{+}} \sum_{i=1}^{m} \sum_{j=1}^{n} c_{i j}^{k} \overline{x_{i j}} \leq \frac{1}{F_{k}^{+}} \sum_{i=1}^{m} \sum_{j=1}^{n} c_{i j}^{k} x_{i j}^{+}, \quad k=\overline{1, r}
$$

and for some indices $k$ we have true the inequality:

$$
\begin{gathered}
\frac{1}{F_{k}^{+}} \sum_{i=1}^{m} \sum_{j=1}^{n} c_{i j}^{k} \overline{x_{i j}} \neq \frac{1}{F_{k}^{+}} \sum_{i=1}^{m} \sum_{j=1}^{n} c_{i j}^{k} x_{i j}^{+} \Rightarrow \\
F(\bar{X})<F\left(X_{L}^{+}\right)
\end{gathered}
$$

The relationship (12), however, contradicts that it is the optimal solution for the model (9). Therefore, our assumption is wrong, and $X_{L}^{+}$is an effective solution to model (4)-(6) what had to be demonstrated.

\section{Method of $Q$-compromise}

This method proposes an approximation of finding the optimal compromise solution for the model (4)-(6), measured by a square compromise distance function [2]. For the proposed model, we will build additional variables such as:

$$
Y_{k}=\frac{1}{F_{k}^{+}} \sum_{i=1}^{m} \sum_{j=1}^{n} c_{i j}^{k} x_{i j}-1, \quad k=1, \ldots, r
$$

Obviously for any admissible solution $(\forall) X \in D$, we have the true relation: $Y_{k} \geq 0$, and only for the optimal solution of the $k$ criterion we will have: $Y_{k}=0$.

We will note:

$$
F(X)=\sum_{k=1}^{r}\left(\frac{1}{F_{k}^{+}} \sum_{i=1}^{m} \sum_{j=1}^{n} c_{i j}^{k} x_{i j}-1\right)^{2}=\sum_{k=1}^{r} Y_{k}^{2}
$$

Function from (14) represents, for $(\forall) X \in D$ the sum of the squares of the normalized distance from the recorded value of the function of the criterion $k$, i.e. $F_{k}(X)$ to the optimal value of this criterion, which is $F_{k}^{+}$.

We will analyse the following model: 
The Method of Synthesis Functions for Solving the Multi-criteria Linear-fractional Transportation Problem with "Bottleneck" Criterion

$$
\begin{aligned}
& \min \sum_{k=1}^{r} Y_{k}^{2} \\
& \text { in condition (5)-(6) }
\end{aligned}
$$

Definition 5. The optimalbasic solution $X_{Q}^{+}$of the model (15) is considered a $Q$-compromise solution for the model (4)-(6).

Theorem 5. Any $Q$ - compromise solution for model (4)-(6) is also efficient for this. Proof. The demonstration we will do it, assuming the contrary.

Let: $X_{Q}^{+}$- Q-compromise solution of the model (4)-(6), which is not efficient for it. Then, there is another feasible solution, according of definition of efficient solution, $\bar{X}=\left\{\overline{x_{i j}}\right\}_{\substack{i=1, m \\ j=1, n}}, \bar{X} \in D$ și $\bar{X} \neq X_{Q}^{+}$such that the relations are true:

$$
\sum_{i=1}^{m} \sum_{j=1}^{n} c_{i j}^{k} \overline{x_{i j}} \leq \sum_{i=1}^{m} \sum_{j=1}^{n} c_{i j}^{k} x_{i j}^{+}, \quad k=\overline{1, r}
$$

and at for some indices $k$ the inequality (16) is strict, i.e. for them is true the relation:

$$
\sum_{i=1}^{m} \sum_{j=1}^{n} c_{i j}^{k} \overline{x_{i j}} \neq \sum_{i=1}^{m} \sum_{j=1}^{n} c_{i j}^{k} x_{i j}^{+} .
$$

In this case we have true the next relationship:

$$
\frac{1}{F_{k}^{+}} \sum_{i=1}^{m} \sum_{j=1}^{n} c_{i j}^{k} \overline{x_{i j}}-1 \leq \frac{1}{F_{k}^{+}} \sum_{i=1}^{m} \sum_{j=1}^{n} c_{i j}^{k} x_{i j}^{+}-1, \quad k=\overline{1, r}
$$

and for some $k$ we have true the inequality:

$$
\begin{gathered}
\frac{1}{F_{k}^{+}} \sum_{i=1}^{m} \sum_{j=1}^{n} c_{i j}^{k} \bar{x}_{i j}-1 \neq \frac{1}{F_{k}^{+}} \sum_{i=1}^{m} \sum_{j=1}^{n} c_{i j}^{k} x_{i j}^{+}-1 \Rightarrow \\
F(\bar{X})<F\left(X_{Q}^{+}\right)
\end{gathered}
$$

The relationship (12), however, contradicts that it is the optimal solution for the model (15). Therefore, our assumption is wrong, and $X_{Q}^{+}$is an efficient solution of model (4)-(6), what had to be demonstrated.

Remark 2. In order to solve the transportation model (15) with quadratic compromise function we can use the quadratic programming method. 
Alexandra Tkacenko

\section{Stages of $L$ - compromise and $Q$ compromise algorithm}

The L-compromise algorithm and the $Q$-compromise algorithm are accomplished by following two similar steps, which are the following:

\section{Phase I}

Step 1. Initially, we will solve $r$ linear transportation models of unicriterial type with the objective functions in (4) on the field of admissible solutions given by the relations (5)-(6);

Step 2. Using the set of optimal solutions from Step 1, the following vector of the objective function recordings is constructed: $F^{+}=\left(F_{1}^{+}, F_{2}^{+}, \ldots, F_{r}^{+}\right)$;

Step 3. We will construct the variables of the following type:

$Y_{k}=\frac{1}{F_{k}^{+}} \sum_{i=1}^{m} \sum_{j=1}^{n} c_{i j}^{k} x_{i j}, \quad k=1, \ldots, r, \quad$ for the $L$-compromise method and $Y_{k}=\frac{1}{F_{k}^{+}} \sum_{i=1}^{m} \sum_{j=1}^{n} c_{i j}^{k} x_{i j}-1, \quad k=1, \ldots, r$ for the $Q$-compromise method;

\section{Phase II}

Step 1. We will construct the objective function: $F(X)=\sum_{k=1}^{r} Y_{k}$ - for the $L$ compromise method and $F(X)=\sum_{k=1}^{r} Y_{k}^{2}$ - for the Q-compromise method;

Step 2. We'll solve the unicriterial transportation model (9), using the $L$ compromise method and model (15), using the $Q$-compromise method.

Remark 3. The $L$-compromise and $Q$-compromise methods, as a rule, record distinct solutions for the model (4)-(6), which leads to the determination of two efficient solutions for it.

\section{The Combinatorial Algorithm}

The algorithm is done in two stages:

\section{Stage 1.}

Step 1. In the time matrix - $T$ of model (3) the time value is ordered in ascending order, thus we will assign to time cells (for identical values of $t$, we will consider 
The Method of Synthesis Functions for Solving the Multi-criteria Linear-fractional Transportation Problem with "Bottleneck" Criterion

different, increasing indices) the indices obtained in result of its ordination. So, the total number of indices will be: $k=m \cdot n$.

Step 2. We will use a required number of cells to place a basic admissible solution, taken in the ascending order of the indices, the other cells will be considered blocked. At a first iteration the number of these cells is at least $k_{1}=m+n-1$; We'll calculate $\overline{\mathrm{T}}_{1}=\max _{i, j}\left\{\bar{t}_{i j} / \bar{x}_{i j}>0\right\}$,

Stage 2. Using the compromise function algorithm we will find the solution of the best compromise for the model (4)-(6), which corresponds to time determined in the first stage. We will go to Step 2 of Stage 1. for unlocking next, the larger cell and higher time respectively.

The algorithm stops when all time cells are unlocked.

\section{Conclusions}

In this paper is developed an integrate multistage procedure to solve the multicriteria fractional transportation problem with "bottleneck" criterion. Applying this algorithm, the decision maker for each possible time value would have determined the best compromise solution for all other criteria From the consideration that selecting the best compromise on a set of efficient solutions takes time, the proposed algorithm is welcome because it saves us from the choice procedure, but it can be used when all criteria are of the same importance. The proposed algorithm is quite necessary and useful, especially when the decision models are of large size and the time for decision making is rather limited.

\section{Example:}

Let be the following 3-criteria problem with 3 supplies and 4 demands.

Supposing that we know the data of unit costs for the first two criteria, which are of course of minimum type, which are the follow:

\begin{tabular}{|c|c|c|c|c|}
\hline \multirow[t]{2}{*}{ Cost $1,2=$} & $\begin{array}{ll}1 & \\
& 5\end{array}$ & 9 & 3 & $\begin{array}{ll}4 & \\
& 10\end{array}$ \\
\hline & $\begin{array}{l}8 \\
\quad 6\end{array}$ & $\begin{array}{l}9 \\
\end{array}$ & $\begin{array}{l}4 \\
\quad 5\end{array}$ & $\begin{array}{ll}6 & \\
& 1\end{array}$ \\
\hline
\end{tabular}


The supply and demand are the next: $A=\{8,19,17\} ; \quad B=\{11,3,14,16\}$.

\section{Solution procedure:}

Time $=$

\begin{tabular}{|l|l|l|l|l|}
\hline 10 & 95 & 73 & 52 & 8 \\
\hline 68 & 66 & 30 & 21 & 19 \\
\hline 37 & 63 & 19 & 23 & 17 \\
\hline 11 & 3 & 14 & 16 & $b_{j} \backslash a_{i}$ \\
\hline
\end{tabular}

By using the efficient Combinatorial Algorithm we have found the following 11 efficient basic solutions:

$$
\begin{aligned}
& X^{1}=\left(x_{11}=8, x_{21}=3, x_{22}=2, x_{23}=14, x_{33}=1, x_{34}=16\right), S^{1}=\left(\frac{176}{68}, \frac{207}{68}, 68\right) ; \\
& X^{2}=\left(x_{11}=8, x_{21}=3, x_{22}=3, x_{24}=13, x_{33}=14, x_{34}=3\right), S^{2}=\left(\frac{164}{68}, \frac{276}{68}, 68\right) ; \\
& X^{3}=\left(x_{11}=6, x_{14}=2, x_{21}=5, x_{23}=14, x_{32}=3, x_{34}=14\right), S^{3}=\left(\frac{178}{68}, \frac{203}{68}, 68\right) ; \\
& X^{4}=\left(x_{11}=8, x_{21}=3, x_{23}=14, x_{24}=2, x_{32}=3, x_{34}=14\right), S^{4}=\left(\frac{172}{68}, \frac{213}{68}, 68\right) ; \\
& X^{5}=\left(x_{11}=8, x_{21}=3, x_{24}=16, x_{32}=3, x_{33}=14\right), S^{5}=\left(\frac{158}{68}, \frac{283}{68}, 68\right) ; \\
& X^{6}=\left(x_{13}=8, x_{21}=11, x_{22}=2, x_{23}=6, x_{32}=1, x_{34}=16\right), S^{6}=\left(\frac{208}{73}, \frac{167}{73}, 73\right) ; \\
& X^{7}=\left(x_{13}=6, x_{14}=2, x_{21}=11, x_{23}=8, x_{32}=3, x_{34}=14\right), S^{7}=\left(\frac{202}{73}, \frac{173}{73}, 73\right) ; \\
& X^{8}=\left(x_{12}=2, x_{13}=6, x_{21}=11, x_{23}=8, x_{32}=1, x_{34}=16\right), S^{8}=\left(\frac{186}{95}, \frac{171}{95}, 95\right) ; \\
& X^{9}=\left(x_{12}=3, x_{13}=5, x_{21}=11, x_{23}=8, x_{33}=1, x_{34}=16\right), S^{9}=\left(\frac{176}{95}, \frac{175}{95}, 95\right) ;
\end{aligned}
$$


The Method of Synthesis Functions for Solving the Multi-criteria Linear-fractional Transportation Problem with "Bottleneck" Criterion

$$
\begin{aligned}
& X^{10}=\left(x_{11}=5, x_{12}=3, x_{21}=6, x_{24}=13, x_{33}=14, x_{34}=3\right), S^{10}=\left(\frac{143}{95}, \frac{265}{95}, 95\right) ; \\
& X^{11}=\left(x_{11}=5, x_{12}=3, x_{21}=6, x_{23}=13, x_{33}=1, x_{34}=16\right), S^{11}=\left(\frac{156}{95}, \frac{200}{95}, 95\right) .
\end{aligned}
$$

From the list of solutions using the best compromise algorithm we highlight the solutions:

$$
\begin{aligned}
& X^{4}=\left(x_{11}=8, x_{21}=3, x_{23}=14, x_{24}=2, x_{32}=3, x_{34}=14\right), S^{4}=\left(\frac{172}{68}, \frac{213}{68}, 68\right) ; \\
& X^{6}=\left(x_{13}=8, x_{21}=11, x_{22}=2, x_{23}=6, x_{32}=1, x_{34}=16\right), S^{6}=\left(\frac{208}{73}, \frac{167}{73}, 73\right) ; \\
& X^{7}=\left(x_{13}=6, x_{14}=2, x_{21}=11, x_{23}=8, x_{32}=3, x_{34}=14\right), S^{7}=\left(\frac{202}{73}, \frac{173}{73}, 73\right) ; \\
& X^{11}=\left(x_{11}=5, x_{12}=3, x_{21}=6, x_{23}=13, x_{33}=1, x_{34}=16\right), S^{11}=\left(\frac{156}{95}, \frac{200}{95}, 95\right) .
\end{aligned}
$$

\section{REFERENCES}

[1]AnejaY.P., Nair K.P.K.(1979), Bicriteria Transportation Problem; Management Sci., V.25, N.1, 73-79;

[2]Diaz Jose A. (1979), Solving Multiobjective Transportation Problems;

Econ-math overview, 15, 62-73;

[3]Garfinkel R.S., Rao N.R.(1971), The Bottleneck Transportation Problems;

N.R.L.Q., 18, 465-472;

[4]Gupta Reeta (1977), Time-cost Transportation Problem; Econ.-Math. Obzor, 4, 431-443;

[5]Isermann H.(1979), The Enumeration of all Efficient Solutions for a Linear Multiple-objective Transportation Problem; N.R.L.Q., V.26, N1, 123-139; [6]Ringuest J.L, Rinks D.B.(1987), Interactive Solutions for the Linear Multiobjective Transportation Problems. European Journal of Operational Research, 32, 96-106;

[7]Sharma S.R., Swarup K.(1977), Transportation Fractional Programming with Respect to Time; Ricerca Operativa, 7(3), 49-58;

[8]Sayed A. Zaki, Abd Allax A. Mousa, Hamdy M. Geneedi, Adel Y.

Elmekawy(2012), Efficient Multiobjective Genetic Algorithm for Solving Transportation, Assignment, and Transsipment Problems; Applied Mathematics, 3, 92-99; 
Alexandra Tkacenko

[9]Tkacenko, A.I.(2014), Multiple Criteria Fuzzy Cost Transportation Model of "Bottleneck" Type. Economic Computation and Economic Cybernetics Studies and Research; ISI Thomson Reuter Serv., V.48, No.2; ASE Publishing, Bucharest, Romania, pp. 215-232;

[10]Waiel F. Abd El-Waheda, Sang M. Lee (2006), Interactive Fuzzy Goal Programming for Multi-objective Transportation Problems; The International Journal of Management Sci., Omega 34, 158 - 166;

[11]Wild Bill Yr., Karwan K.R., Karwan M.H.(1994), The Multiple

“Bottleneck” Transportation Problem; Computer Ops. Res., Vol.20, No.3, pp. 261-274. 